\title{
Dielectronic recombination rate coefficients of fluorine-like nickel
}

\author{
Shu-Xing Wang ${ }^{1,2}$, Zhong-Kui Huang ${ }^{3}$, Wei-Qiang $\mathrm{Wen}^{3}$, Chong-Yang $\mathrm{Chen}^{4}$, Stefan Schippers ${ }^{5}$, Xin $\mathrm{Xu}^{1,2}$, \\ Shahid Sardar ${ }^{1,2}$, Nadir Khan ${ }^{3}$, Han-Bing Wang ${ }^{3}$, Li-Jun Dou ${ }^{3}$, Sultan Mahmood ${ }^{3,6}$, Dong-Mei Zhao ${ }^{3}$, \\ Xiao-Long $\mathrm{Zhu}^{3}$, Li-Jun $\mathrm{Mao}^{3}$, Xiao-Ming $\mathrm{Ma}^{3}$, Jie $\mathrm{Li}^{3}$, Mei-Tang Tang ${ }^{3}$, Rui-Shi $\mathrm{Mao}^{3}$, Da-Yu Yin ${ }^{3}$, \\ You-Jin Yuan ${ }^{3}$, Jian-Cheng Yang ${ }^{3}$, Ying-Long Shi ${ }^{7}$, Chen-Zhong Dong ${ }^{8}$, Xin-Wen $\mathrm{Ma}^{3}$, and Lin-Fan Zhu ${ }^{1,2}$ \\ ${ }^{1}$ Hefei National Laboratory for Physical Sciences at Microscale and Department of Modern Physics, University of Science and \\ Technology of China, Hefei, Anhui 230026, PR China \\ e-mail: lfzhu@ustc.edu.cn \\ 2 Synergetic Innovation Center of Quantum Information and Quantum Physics, University of Science and Technology of China, \\ Hefei, Anhui 230026, PR China \\ 3 Institute of Modern Physics, Chinese Academy of Sciences, Lanzhou, Gansu 730000, PR China \\ e-mail: x.ma@impcas.ac.cn, wenweiqiang@impcas.ac.cn \\ ${ }^{4}$ Shanghai EBIT Laboratory, Institute of Modern Physics, Fudan University, and the Key Laboratory of Applied Ion Beam Physics, \\ Chinese Ministry of Education, Shanghai 200433, PR China \\ e-mail: chychen@fudan.edu.cn \\ 5 I. Physikalisches Institut, Justus-Liebig-Universität Gießen, Heinrich-Buff-Ring 16, 35392 Giessen, Germany \\ 6 Physics Division, PINSTECH, Nilore, Islamabad 45650, Pakistan \\ 7 Institute for Fundamental Physics, Tianshui Normal University, Tianshui, Gansu 741000, PR China \\ 8 Key Laboratory of Atomic and Molecular Physics and Functional Materials of Gansu Province, College of Physics and Electronic \\ Engineering, Northwest Normal University, Lanzhou, Gansu 730070, PR China
}

Received 9 April 2019 / Accepted 4 June 2019

\begin{abstract}
Electron-ion recombination rate coefficients for fluorine-like nickel ions have been measured by employing the merged-beam technique at the cooler storage ring CSRm at the Institute of Modern Physics in Lanzhou, China. The measured spectrum covers the energy range of $0-160 \mathrm{eV}$, including all the dielectronic recombination (DR) resonances associated with $\Delta N=0$ core excitations. The DR cross sections in this energy range were calculated by a relativistic configuration interaction method using the flexible atomic code (FAC). Radiative recombination (RR) cross sections were obtained from a modified version of the semi-classical Bethe \& Salpeter (1957, Quantum Mechanics of One- and Two-Electron 56 Systems (Springer)) formula for hydrogenic ions. The comparison between the measurement and the calculation shows that the present theoretical model still needs to be improved at low collision energies. Temperature dependent plasma recombination rate coefficients were derived from the measured DR rate coefficients in the temperature range of $10^{3}-10^{8} \mathrm{~K}$ and compared with the presently calculated result as well as previous available data in the literature. The experimentally derived data agree well with the theoretical calculations for temperatures where $\mathrm{Ni}^{19+}$ ions form in collisionally ionized plasmas. At lower temperatures typical for photo-ionized plasmas, discrepancies are found beyond the experimental uncertainty, which can be attributed to the disagreement between the measurement and the calculation of the low-lying DR resonances. The present experimental result benchmarks the plasma DR rate coefficients, in particular for temperatures below $10^{5} \mathrm{~K}$ where the $\Delta N=0 \mathrm{DR}$ resonances dominate.
\end{abstract}

Key words. atomic data - atomic processes - plasmas

\section{Introduction}

X-ray astronomy is primarily an observational science performed by detecting photons. Various types of spaced-based observatories, such as the Solar Maximum Mission (NASA), the Solar and Heliospheric Observatory (NASA), Chandra (NASA) and XMM-Newton (ESA), have been launched to collect highresolution X-ray data (Beiersdorfer 2003). It is well known that more than $95 \%$ of the visible matter in the universe is made of plasma, a mixture of electrons, atoms and positive and negative ions (Beyer \& Shevelko 2003). Electron-ion collision processes that can result in line emissions, particularly, radiative recombination (RR) and dielectronic recombination (DR), are consequently important sources of radiation in astrophysical plasmas. In addition electron-ion recombination rate coefficients and electron impact ionization (EII) or photoioniza- tion (PI) cross sections are essential for deducing the charge state distribution (CSD) in a plasma. Accurate CSD data are required for determining further properties of a plasma, such as thermal structure and elemental abundances (Beiersdorfer 2003; Savin 2007). Uncertainties in the DR rate coefficients will carry through to the calculations of the ionization balance and to the inferred relative abundances and the first ionization potential factors (Savin \& Laming 2002). Thus, reliable electron-ion recombination data are essential for astrophysicists and plasma modelers.

Dielectronic recombination is a two-step resonant process. It begins when a free electron collisionally excites an ion and is simultaneously captured into a Rydberg level. The doubly excited autoionizing states can either autoionize or decay radiatively to reduce its total energy below the ionization limit, which completes the DR process. RR is the time-inverse 
process of direct PI. The significance of DR was first recognized by Burgess (1964) as an important recombination mechanism in the solar corona, dominating over the direct RR channel in hightemperature plasmas. Most recombination rate coefficients of highly charged ions (HCIs) available in the literature are derived from theoretical calculations. However, the theoretical prediction of the DR resonance positions into a low- $n$ Rydberg level is still a challenging task due to the strong electron correlation between the captured and the core electrons (Savin \& Laming 2002). In addition, tractable calculations are usually based on reasonable approximations since an infinite number of states should be taken into account with a finite basis expansion (Hahn 1993). Spectroscopically observed core transition energies are usually used to improve the accuracy of the calculated DR resonance positions associated with the $\Delta N=0$ core excitations ( $\mathrm{Gu} 2003$ ). The currently available atomic codes still cannot produce DR rate coefficients with a sufficient precision at low electron-ion collision energies. Uncertainties of the resonance positions at low electron-ion collision energies can translate into huge discrepancies in the derived temperature dependent plasma rate coefficients (Schippers et al. 2004). Recent experimental studies have also shown that the previously calculated low-temperature DR rate coefficients are not reliable (Schippers 2009; Huang et al. 2018). Therefore, accurate experimental DR rate coefficients of astrophysical relevant HCIs are required to benchmark different theoretical methods to produce more reliable electron-ion recombination data.

Nickel is the second most abundant iron-peak heavy element (Feldman \& Laming 2000; Asplund et al. 2009) in the solar system which also reflects the element abundance in the universe. Line emission originating from nickel ions has been widely observed from a variety of cosmic objects. Examples are the high-resolution X-ray spectra from two solar flares observed with Flat Crystal Spectrometer on board the Solar Maximum Mission (Phillips et al. 1982) or the Fe/Ni features observed during flares by the broadband RHESSI spectrometer (Phillips 2004). In addition, the unusual $\mathrm{Ni} / \mathrm{Fe}$ abundance ratio in the Crab nebula filaments was found to exceed the solar ratio by an order of magnitude (Henry 1984). Summaries of the observed spectral lines associated with nickel ions and with astrophysical abundant elements have been given by Doschek \& Feldman (2010) in a topical review. Radiation associated with DR of F-like ions via the fine structure core excitations provides the basis for new classes of electron temperature and density diagnostics in photoionized plasma (Savin et al. 1997, 1999). In addition to astrophysical plasmas, $\mathrm{Ni}$ is also an important heavy impurity in tokamak plasmas. Earlier attempts to model the fractional abundances were limited by the unreliable calculated DR rate coefficients (Group 1980). Here we present the absolute DR rate coefficients of F-like nickel ions from a heavy ion storage ring measurement as well as from the theoretical calculation with the flexible atomic code (FAC; Gu 2008).

Dielectronic recombination resonances associated with $\Delta N=0$ core excitations of F-like ${ }^{58} \mathrm{Ni}^{19+}$ ions can be expressed as:

$$
\begin{aligned}
& { }^{58} \mathrm{Ni}^{19+}\left(2 \mathrm{~s}^{2} 2 \mathrm{p}^{5}\left[{ }^{2} \mathrm{P}_{3 / 2}\right]\right)+\mathrm{e}^{-} \rightarrow \\
& \left\{\begin{array}{c}
{ }^{58} \mathrm{Ni}^{18+}\left(2 \mathrm{~s}^{2} 2 \mathrm{p}^{5}\left[{ }^{2} \mathrm{P}_{1 / 2}\right] \mathrm{nl}\right)^{* *} \rightarrow{ }^{58} \mathrm{Ni}^{18+}+\gamma ; \\
{ }^{58} \mathrm{Ni}^{18+}\left(2 \mathrm{~s} 2 \mathrm{p}^{6}\left[{ }^{2} \mathrm{~S}_{1 / 2}\right] \mathrm{nl}\right){ }^{* *} \rightarrow{ }^{58} \mathrm{Ni}^{18+}+\gamma .
\end{array}\right.
\end{aligned}
$$

The associated excitation energies for $2 \mathrm{~s}^{2} 2 \mathrm{p}^{5}\left[{ }^{2} \mathrm{P}_{3 / 2}\right] \rightarrow$ $2 \mathrm{~s}^{2} 2 \mathrm{p}^{5}\left[{ }^{2} \mathrm{P}_{1 / 2}\right]$ and $2 \mathrm{~s}^{2} 2 \mathrm{p}^{5}\left[{ }^{2} \mathrm{P}_{3 / 2}\right] \rightarrow 2 \mathrm{~s} 2 \mathrm{p}^{6}\left[{ }^{2} \mathrm{~S}_{1 / 2}\right]$ are $17.8486 \mathrm{eV}$ and $149.054 \mathrm{eV}$, respectively (Kramida et al. 2018). The first channel involves the fine-structure core excitation of a $2 \mathrm{p}_{1 / 2}$ electron to the $2 p_{3 / 2}$ subshell and the second channel involves the core excitation of a $2 \mathrm{~s}_{1 / 2}$ electron to the $2 \mathrm{p}_{3 / 2}$ subshell. DR resonances associated with the fine-structure core transition occurs at low collision energies, leading to an important contribution to the plasma rate coefficients for a photoionized gas. In addition, the respective autoionizing states will stabilize by radiative decay of the captured electron since the radiative decay of the inner electron is electric-dipole forbidden.

Storage rings equipped with an electron cooler are effectively the only available tools for measuring accurate DR rate coefficients of HCIs at low electron-ion collision energies. A large number of DR experiments have been carried out at storage rings, for example the Test Storage Ring (TSR) at Max-PlanckInstitut für Kernphysik (MPIK) in Heidelberg (Schippers 2015), the Experimental Storage Ring (ESR) at GSI Helmholtzzentrum für Schwerionenforschung (GSI) in Darmstadt (Brandau et al. 2015) and CRYRING at Manne Siegbahn Laboratory (MSL) in Stockholm (Schuch \& Böhm 2007). More details about DR experiments at the storage rings can be found in recent reviews (Schuch \& Böhm 2007; Müller 2008; Schippers 2015) and the references therein. The only storage-ring DR experiments with F-like ions available in literature are the ones with $\mathrm{Se}^{25+}$ (Lampert et al. 1996) and $\mathrm{Fe}^{17+}$ (Savin et al. 1997, 1999). Previously available $\mathrm{DR}$ data for $\mathrm{Ni}$ ions from storage ring measurement are data for Li-like $\mathrm{Ni}^{25+}$ (Schippers et al. 2000) and Na-like $\mathrm{Ni}^{17+}$ (Fogle et al. 2003a,b).

Benchmark DR data for L-shell ions are widely required in astrophysical applications, however, only a few experiments have been performed. Here we report on a storage ring measurement of the electron-ion recombination rate coefficients of $\mathrm{F}$-like $\mathrm{Ni}^{19+}$ comprising all DR resonances associated with all the $\Delta N=0$ core excitations. The paper is structured as follows: In Sect. 2, the experimental procedures and data analysis are described; in Sect. 3, a brief introduction to the present theoretical calculation is given; merged-beam DR rate coefficients as well as the plasma rate coefficients are presented and discussed in Sect. 4; finally we summarize the results in Sect. 5 as a conclusion.

\section{Experiment and data analysis}

The experiment was performed at the Main Cooler Storage Ring (CSRm) at the Institute of Modern Physics in Lanzhou, China. A detailed description of the DR experimental setup and procedures at the CSRm were already given in our previous publications (Huang et al. 2015, 2018; Wang et al. 2018). Here we briefly describe the DR experiment with F-like ${ }^{58} \mathrm{Ni}^{19+}$ at the CSRm.

The ${ }^{58} \mathrm{Ni}^{19+}$ ions were produced from a superconducting electron cyclotron resonance (ECR) ion source, accelerated by a sector focused cyclotron (SFC) and then injected into the $\mathrm{CSRm}$ at an energy of $6.15 \mathrm{MeV} / \mathrm{u}$. Every injection pulse of ions was sufficient to maintain a maximum ion-beam current of about $80 \mu \mathrm{A}$, corresponding to $3.7 \times 10^{8}$ stored ions in the ring. The circulating ion beam was first electron-cooled for about $2 \mathrm{~s}$ by immersing it in a magnetically expanded cold electron beam after every injection (Danared 1993). The magnetic fields applied at the cathode and the cooler section were $125 \mathrm{mT}$ and $39 \mathrm{mT}$, respectively. The electron beam was used as the electron target in the recombination experiment as well. In the cooler section, the primary ion beam was merged with the electron beam over an effective interaction length $L$ of $4.0 \mathrm{~m}$. The expanded diameter of the electron beam was about $62 \mathrm{~mm}$ and the electron density in the cooler section was about $7.1 \times 10^{6} \mathrm{~cm}^{-3}$. The recombined ions formed in the cooler section 
were separated from the primary ion beam in the first dipole magnet downstream from the electron cooler and detected by a movable scintillation particle detector (YAP: Ce+PMT) with nearly $100 \%$ detection efficiency (Wen et al. 2013).

The electron energy was set at $3.507 \mathrm{keV}$ during electron cooling, corresponding to zero electron-ion collision energy in the center of mass frame. Offset voltages were applied to the cathode voltage by a suitably designed detuning system to obtain nonzero collision energies. In addition, a direct current current transformer (DCCT) was used to monitor the real-time ion beam current and the storage beam lifetime was estimated accordingly. Two ion beam position monitors (BPMs) and one electron BPM were utilized to monitor the spatial overlap of the electron beam and the ion beam in the cooling section. Schottky-noise signals were recorded and analyzed by a Tektronix RSA3408 spectrum analyzer to monitor the revolution frequency and the momentum spread of the ions. The latter was about $\Delta \mathrm{p} / \mathrm{p} \sim 2 \times 10^{-4}$ during the experiment.

The electron-ion collision energy in the center of mass frame was calculated using

$E_{\text {rel }}=\sqrt{m_{\mathrm{e}}^{2} c^{4}+m_{\mathrm{i}}^{2} c^{4}+2 m_{\mathrm{e}} m_{\mathrm{i}} \gamma_{\mathrm{e}} \gamma_{\mathrm{i}} c^{4}\left(1-\beta_{\mathrm{e}} \beta_{\mathrm{i}} \cos \theta\right)}-m_{\mathrm{e}} c^{2}-m_{\mathrm{i}} c^{2}$

where $m_{\mathrm{e}}$ and $m_{\mathrm{i}}$ are respectively the electron and ion rest mass. $c$ is the speed of light, and $\gamma_{\mathrm{e}}$ and $\gamma_{\mathrm{i}}$ respectively denote the Lorentz factors of electron and ion beams. The angle $\theta$ between electron and ion beam is safely considered as zero in the present experiment. Space charge effects were taken into account. Drag force effects were found to be negligible. The calculated collision energies were then recalibrated by multiplying them by a factor of 0.98 to match the known $2 \mathrm{~s} 2 \mathrm{p}^{6}\left[{ }^{2} \mathrm{~S}_{1 / 2}\right]$ nl series limit.

The absolute electron-ion recombination rate coefficient as a function of the collision energy can be deduced from the energy dependent detector count rate $\mathrm{R}(\mathrm{E})$ as:

$\alpha(E)=\frac{R(E)}{N_{\mathrm{i}} n_{\mathrm{e}}\left(1-\beta_{\mathrm{e}} \beta_{\mathrm{i}}\right)} \frac{C}{L}$.

Here, $N_{\mathrm{i}}$ is the number of the stored ions in the ring, $n_{\mathrm{e}}$ is the electron density, $C=161.0 \mathrm{~m}$ and $L=4.0 \mathrm{~m}$ denote the circumference of the ring and the length of the effective interaction section, respectively; and $v_{\mathrm{e}}=\beta_{\mathrm{e}} c$ and $v_{\mathrm{i}}=\beta_{\mathrm{i}} c$ are the velocities of electron and ion beams in the laboratory frame.

The measurement covers the energy range $0-160 \mathrm{eV}$ corresponding to detuning voltages of $0-1300 \mathrm{~V}$. The uncertainty of the measured rate coefficient is estimated to be about $30 \%$ at a $1 \sigma$ confidence level, including a $10 \%$ uncertainty for counting statistics, the electron and ion beam current, and the electron-ion interaction length; an uncertainty of $15 \%$ due to the background subtraction; and an uncertainty of $25 \%$ for the electron density in the cooler section. It should be noted that the experiment was carried out with six different detuning timing scales to ensure that the increasing detuning voltages can be applied to the cathode effectively. The rate coefficients measured with the last two timescales, corresponding to the collision energy range of 110$160 \mathrm{eV}$, show a little more fluctuations due to the long detuning time during the measurement.

\section{Theory}

For a better understanding of the present measured DR spectrum of F-like nickel, DR resonance strengths were calculated using the isolated resonance approximation (IRA) with the distortedwave method based on the Dirac equation. Atomic energy levels, auto-ionization and radiative decay rates were calculated with a relativistic atomic-structure program, the FAC (Gu 2003, 2008). Since the calculated energy levels may have an uncertainty of a few $\mathrm{eV}$, the resonance energies were empirically adjusted with the core excitation energies from the NIST atomic database (Kramida et al. 2018). These adjustments were necessary for DR processes via $\Delta N=0$ core excitations to obtain more reliable low-lying DR resonances from the calculation.

The DR process was treated as two independent steps in the calculation. The first step is dielectronic capture (DC) which is the time inverse of auto-ionization (AI). The doubly-excited autoionizing intermediate state can either auto-ionize or decay radiatively thereby completing the DR process. As a result, the DR cross section via a particular doubly-excited state is the product of the DC cross section and the branching ratio for radiative stabilization. The DC cross sections are related to the AI rates through the principle of detailed balance. Since DC is a resonant process it only occurs when an energy matching condition is fulfilled. The resonances are usually much narrower than the experimental electron energy spread, except at low collision energies (see below). Therefore, in most cases, it is sufficient to characterize the DR resonances by their strengths, which are the integrated cross sections over energy. The DC strength can be written as:

$S_{\mathrm{DC}}=\frac{g_{\mathrm{i}}}{2 g_{\mathrm{f}}} \frac{\pi^{2}}{E_{\mathrm{if}}} A^{a}$

where $g_{\mathrm{i}}$ and $g_{\mathrm{f}}$ are the statistical weights of the initial state and final autoionizing state after DC, respectively; $E_{\text {if }}$ is the resonance energy; and $A^{a}$ is the AI strength. The radiative branching ratio can be approximately expressed as

$B(i)=\frac{\sum_{k} A_{i k}^{r}+\sum_{k^{\prime}} A_{i k^{\prime}}^{r} \cdot B\left(k^{\prime}\right)}{\sum_{k} A_{i k}^{r}+\sum_{j} A_{i j}^{a}}$

where $A_{\mathrm{if}}^{r}$ denotes the radiative decay rate from state $i$ to $f ; k$ and $k^{\prime}$ represent the states below the ionization threshold and the states that may further autoionize, respectively; and $A_{i j}^{a}$ denotes the auto-ionization rate from state $i$ to state $j$. Cascade processes to autoionizing states are taken into account using an iterative procedure to calculate the radiative stabilizing branching ratio (Savin 2007).

\section{Results and discussion}

In order to explain our results clearly, we present the mergedbeam and plasma recombination rate coefficients separately. The merged-beam recombination spectra present the resonance features as a function of electron-ion collision energies. The plasma recombination rate coefficients present the plasma temperature dependent averaged DR rate coefficients. We will discuss the results in detail in the following two sections.

\subsection{Merged-beam recombination rate coefficients}

The black filled circles in Fig. 1 represent the merged-beam rate coefficients of F-like $\mathrm{Ni}$ recombined into Ne-like Ni. The measurement covers the energy range of $0-160 \mathrm{eV}$, including all the DR resonances associated with the $2 \mathrm{~s}^{2} 2 \mathrm{p}^{5}\left[{ }^{2} \mathrm{P}_{3 / 2}\right] \rightarrow$ $2 \mathrm{~s}^{2} 2 \mathrm{p}^{5}\left[{ }^{2} \mathrm{P}_{1 / 2}\right]$ and $2 \mathrm{~s}^{2} 2 \mathrm{p}^{5}\left[{ }^{2} \mathrm{P}_{3 / 2}\right] \rightarrow 2 \mathrm{~s} 2 \mathrm{p}^{6}\left[{ }^{2} \mathrm{~S}_{1 / 2}\right]$ core excitations. The vertical bars in Fig. 1 are the resonance positions estimated by the following Rydberg formula:

$E_{\mathrm{res}}(n)=E_{\mathrm{exc}}-R \frac{q^{2}}{n^{2}}$ 


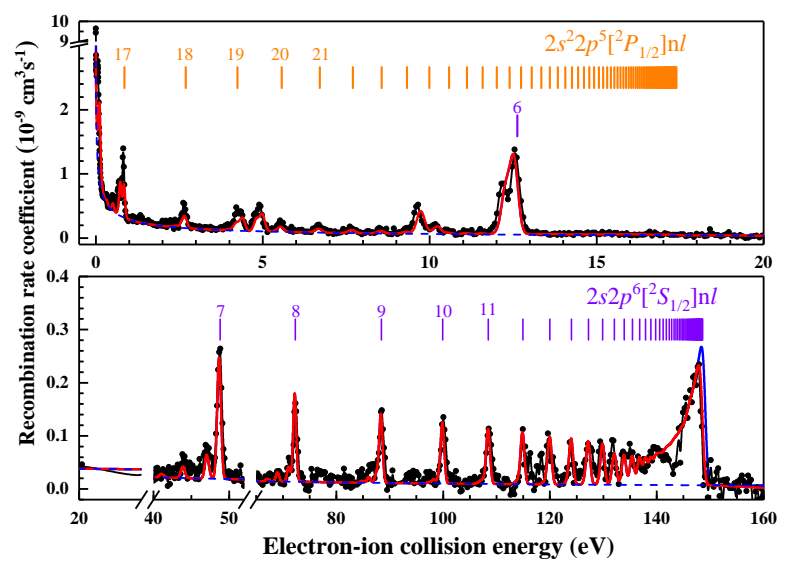

Fig. 1. Measured and calculated recombination rate coefficients of F-like Ni. The experimental result (connected filled circles) covers the energy range of $0-160 \mathrm{eV}$. The red solid line denotes the theoretically calculated recombination spectrum and the blue dashed line shows the RR background. The vertical bars are the resonance positions of $2 \mathrm{~s}^{2} 2 \mathrm{p}^{5}\left[^{2} \mathrm{P}_{1 / 2}\right] \mathrm{nl}$ and $2 \mathrm{~s} 2 \mathrm{p}^{6}\left[{ }^{2} \mathrm{~S}_{1 / 2}\right] \mathrm{nl}$ series estimated by the Rydberg formula. The blue solid line accounts for the theoretical DR rate coefficients of the captured electron with principal quantum number up to $n_{\max }=1000$.

where the Rydberg constant $R \approx 13.60569 \mathrm{eV}$, the primary ion charge state $q=19, E_{\text {exc }}$ is the core transition energy, and $n$ denotes the principal quantum number of the captured electron. The formula works well for the free electron recombined into high Rydberg levels but is inadequate for low $n$ levels where fine structure effects dominate the DR resonance structure. Recombined Rydberg electrons with the principal quantum numbers $n>n_{\text {cutoff }}$ will be field-ionized in the dipole bending magnets of the storage ring before being detected. As a result, the fieldionized ions will not reach the detector. Here, $n_{\text {cutoff }}=96$ as calculated by (Fogle et al. 2005):

$n_{\text {cutoff }} \approx\left(6.2 \times 10^{8} \mathrm{~V} \mathrm{~cm}^{-1} \frac{Q^{3}}{v_{\mathrm{i}} B}\right)^{1 / 4}$.

The red solid line in Fig. 1 is the theoretically calculated recombination spectrum. The recombination cross sections were transformed into rate coefficients according to the formula:

$\alpha(E)=\int_{-\infty}^{+\infty} \sigma(v) v f\left(v, T_{\|}, T_{\perp}\right) \mathrm{d}^{3} v$,

where $f\left(v, T_{\|}, T_{\perp}\right)$ is the anisotropic electron velocity distribution. The characteristic parallel and perpendicular electron temperatures were obtained by fitting the low-energy spectrum of $0-1.2 \mathrm{eV}$ as shown in Fig. 2. In the fitting, we use a Lorentzian function multiplied by a factor of $E_{\text {res }} / E$ as the line shape for the first resonance (Schippers et al. 2004). The cross sections for other resonances are treated as delta functions since their natural line widths are much narrower than the present experimental energy spread. The fitted width for the first resonance at about $80 \mathrm{meV}$ is $51(5) \mathrm{meV}$, comparable to the FAC calculated $60 \mathrm{meV}$. The fitted parallel and perpendicular electron temperatures are $k_{\mathrm{B}} T_{\|}=0.56(0.05) \mathrm{meV}$ and $k_{\mathrm{B}} T_{\perp}=23(1) \mathrm{meV}$, respectively. The calculated DR cross sections account for the principal quantum number of the captured electron up to $n=n_{\text {cutoff }}(96)$. The calculated DR cross sections for the $2 s^{2} 2 p^{5}\left[{ }^{2} \mathrm{P}_{1 / 2}\right] \mathrm{nl}$ and the $2 \mathrm{~s} 2 \mathrm{p}^{6}\left[{ }^{2} \mathrm{~S}_{1 / 2}\right] \mathrm{nl}$ series were then extrapolated to $n_{\max }=1000$ to remedy the field ionization effect for the derivation of the plasma

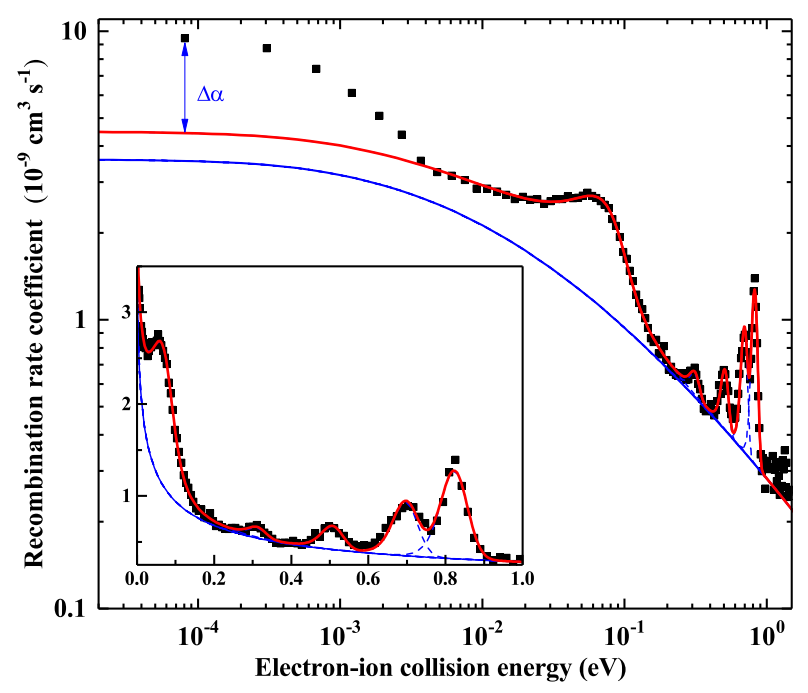

Fig. 2. Fitted curve (red solid line) of the measured DR spectrum below $1.2 \mathrm{eV}$. The blue solid line indicates the fitted RR background and the blue dashed lines depict the line shape of each single peak. The fitted parallel and perpendicular electron temperatures are $k_{\mathrm{B}} T_{\|}=$ $0.56(0.05) \mathrm{meV}$ and $k_{\mathrm{B}} T_{\perp}=23(1) \mathrm{meV}$, respectively. $\Delta \alpha$ denotes the recombination rate enhancement at very low energies (below $100 \mathrm{meV}$ ) which is the artifact of the merged-beam method (Gwinner et al. 2000).

rate coefficient below. The RR background was obtained with a modified version of the semi-classical Bethe \& Salpeter (1957) formula for the hydrogenic RR cross section (Böhm et al. 2001). A factor of 1.57 was applied to modify the RR rate coefficients for F-like Ni according to the fit. At energies below $4 \mathrm{meV}$, the measured rate coefficients demonstrate an enhancement $(\Delta \alpha)$ of a factor of about 2.1 over the fitted rate coefficients. The detailed discussion of the rate coefficient enhancement is beyond the scope of this paper.

Figures 3 and 4 present the detailed comparison between the measured DR rate coefficients and the FAC calculations below $20 \mathrm{eV}$. The comparison shows that the low-energy resonance positions and strengths are still not well produced by the present calculation. We fitted the experimentally resolved peaks to extract the resonance energies and strengths. The fitted values are listed in Table A. 1 for comparison with the FAC calculated values. The various $2 \mathrm{~s} 2 \mathrm{p}^{6}\left[{ }^{2} \mathrm{~S}_{1 / 2}\right] 61$ fine-structure levels range from the first peak at about $80 \mathrm{meV}$ to the features at around $12 \mathrm{eV}$. An accurate prediction of the $2 \mathrm{~s} 2 \mathrm{p}^{6}\left[{ }^{2} \mathrm{~S}_{1 / 2}\right] 61$ resonance positions is difficult due to the strong interaction between the captured electron with the core. In particular, the calculated $2 \mathrm{~s} 2 \mathrm{p}^{6}\left[{ }^{2} \mathrm{~S}_{1 / 2}\right] 61(J=1)$ resonance position is about $50 \%$ larger than the experimentally extracted value. The $2 \mathrm{~s}^{2} 2 \mathrm{p}^{5}\left[{ }^{2} \mathrm{P}_{1 / 2}\right] \operatorname{nl}(n=17-20)$ resonances are also not accurately produced by the calculation. The experimentally extracted strengths for the $2 \mathrm{~s}^{2} 2 \mathrm{p}^{5}\left[{ }^{2} \mathrm{P}_{1 / 2}\right] 171$ resonances are more than a factor of 1.8 over the predicted ones. Instead of a pileup at the $\left.2 s^{2} 2 p^{5}{ }^{2} \mathrm{P}_{1 / 2}\right] \mathrm{nl}$ series limit at about $17 \mathrm{eV}$, a stair-like structure is observed because the optical forbidden $2 \mathrm{~s}^{2} 2 \mathrm{p}^{5}\left[{ }^{2} \mathrm{P}_{3 / 2}\right] \rightarrow$ $2 s^{2} 2 p^{5}\left[{ }^{2} \mathrm{P}_{1 / 2}\right]$ core transitions suppresses the recombination with the electron into a high Rydberg level. At higher energies, the rate coefficients are dominated by the recombination via $2 \mathrm{~s} 2 \mathrm{p}^{6}\left[{ }^{2} \mathrm{~S}_{1 / 2}\right] \operatorname{nl}(n \geq 7)$ intermediate states. We estimate that there will be an additional uncertainty for the extracted energies and strengths from the very weak resonances (marked as presuper d in Table A.1). The measured rate coefficients agree with the theoretical calculation on a reasonable level. The measured 


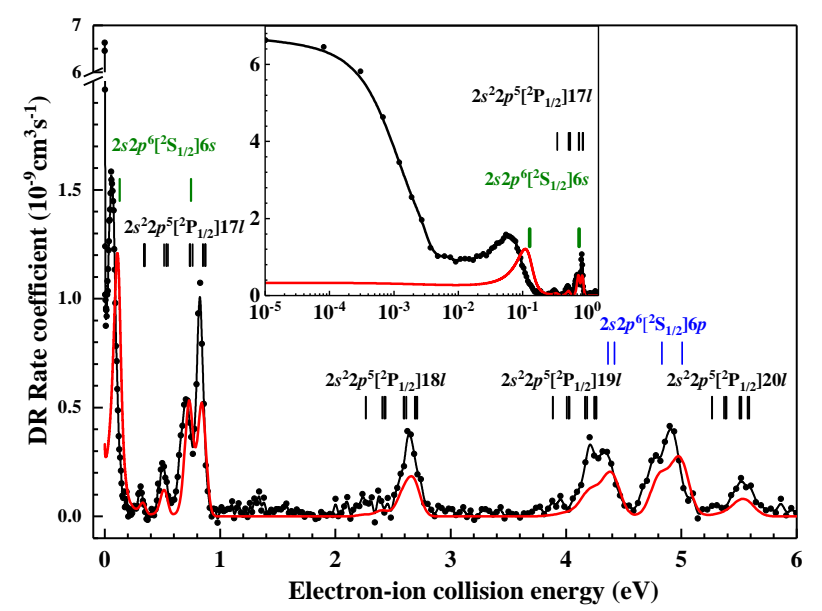

Fig. 3. Detailed comparison between the measured spectrum (black dotconnected line) and the theoretical spectrum calculated by FAC (red solid line). The vertical bars denote the calculated resonance positions of the corresponding intermediate states.

spectrum beyond $110 \mathrm{eV}$ cannot be fitted reasonably because of the fluctuations there.

\subsection{Plasma recombination rate coefficients}

Cosmic plasmas are usually divided into two broad classes (Savin 2007): collisionally ionized plasmas (e.g. stars, galaxies and supernova remnants) and photoionized plasmas (e.g. planetary nebulae, X-ray binaries and active galactic nuclei). For convenient use of our experimental data in astrophysics and plasma modeling, temperature dependent plasma recombination rate coefficients were derived from the measured merged-beam DR data. The DR rate coefficients were convolved with the Maxwell-Boltzmann electron energy distribution (Savin et al. 1999; Orban et al. 2009):

$\alpha\left(T_{\mathrm{e}}\right)=\int \alpha(E) f\left(E, T_{\mathrm{e}}\right) \mathrm{d} E$,

where $T_{\mathrm{e}}$ is the plasma temperature, $\alpha(E)$ is the energy dependent DR rate coefficients, $f\left(E, T_{\mathrm{e}}\right)$ is the electron energy distribution (Böhm et al. 2001):

$f\left(E, T_{\mathrm{e}}\right)=\frac{2 E^{1 / 2}}{\pi^{1 / 2}\left(k T_{\mathrm{e}}\right)^{3 / 2}} \exp \left(-\frac{E}{k T_{\mathrm{e}}}\right)$.

The experimental recombination rate coefficients at very low collision energies suffer from an enhancement, which is not expected in a plasma. We use the extracted resonance energies and strengths below $1 \mathrm{eV}$ to obtain the corresponding plasma rate coefficients (Schippers et al. 2004). The measured DR rate coefficients beyond $110 \mathrm{eV}$ are replaced by the theoretical data to cancel the field ionization effect and the influence of the fluctuations. Since the field ionization effect is not expected in a cosmic plasma, the present calculation accounted for principal quantum numbers of up to $n_{\max }=1000$.

Figure 5 presents the experimentally derived plasma rate coefficients and the present FAC calculated data as well as the previous calculations available in the literature. The temperature covers the ranges where $\mathrm{Ni}^{19+}$ ions form in photoionized and collisionally ionized plasmas. The violet dashed line is the data for $\Delta N=0$ DR by (Gu 2003) calculated with FAC code. The blue solid line is the data calculated with the AUTOSTRUCTURE

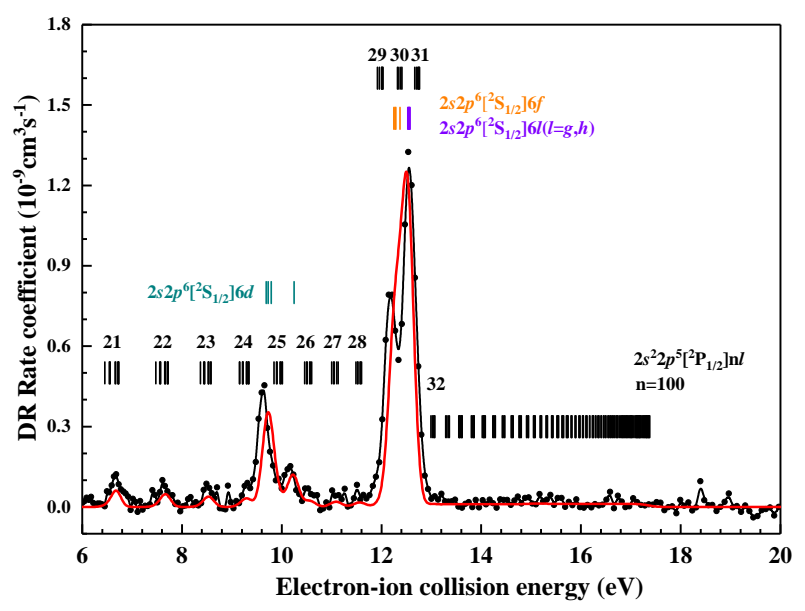

Fig. 4. Same as Fig. 3, but for the energy range of $6-20 \mathrm{eV}$.

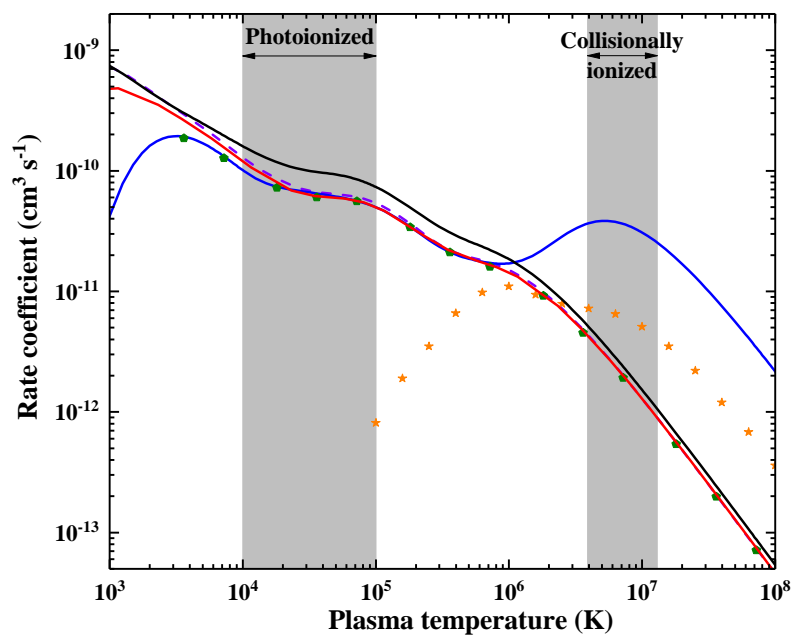

Fig. 5. Experimentally derived plasma recombination rate coefficients (black solid line) compared with the present and previous calculated data. The red solid line is the plasma rate coefficients derived from the presently calculated DR rate coefficients, the violet dashed line is the calculated data by $\mathrm{Gu}$ (2003), the blue solid line denotes the calculation by Zatsarinny et al. (2006) with the AUTOSTRUCTURE code, the orange stars are the previous recommended DR data (Jacobs et al. 1980), and the green pentagons are the data from the Open-ADAS website.

code which includes the $\Delta N=0$ and $\Delta N=1$ DR rate coefficients (Zatsarinny et al. 2006), the green pentagons are the data calculated by the AUTOSTRUCTURE code with only $\Delta N=$ $0 \mathrm{DR}$ from the Open-ADAS ${ }^{1}$. The orange stars are the previous recommended data which included the $\Delta N=0$ and $\Delta N=1 \mathrm{DR}$ rate but the DR via the $2 \mathrm{~s}^{2} 2 \mathrm{p}^{5}\left[{ }^{2} \mathrm{P}_{3 / 2}\right] \rightarrow 2 \mathrm{~s}^{2} 2 \mathrm{p}^{5}\left[{ }^{2} \mathrm{P}_{1 / 2}\right]$ core excitation was not taken into account (Jacobs et al. 1980). The Z-scaled plasma rate coefficients of Mazzotta et al. (1998) and the extrapolated plasma rate coefficients of Shull \& Van Steenberg (1982) are not included; the reasons for not using them are given by $(\mathrm{Gu} 2003)$.

At temperatures below $10^{4} \mathrm{~K}$, the calculated rate coefficients are found to be $20 \%-30 \%$ lower than the measured data. At temperatures around $3 \times 10^{4} \mathrm{~K}$, which are typical of the photoionized plasmas, the calculated rate coefficients are found to be $40 \%$ lower than the experimentally derived values. The mismatch of the $2 s 2 p^{6}\left[{ }^{2} S_{1 / 2}\right] 61$ resonances and the underestimate of the

1 http://open.adas.ac.uk/ 
Table 1. Fitted parameters for the resonant recombination channels derived from the experimental and calculated rate coefficients.

\begin{tabular}{lcccc}
\hline \hline \multirow{2}{*}{ No. } & \multicolumn{2}{c}{ Experiment } & \multicolumn{2}{c}{ FAC calculation } \\
\cline { 2 - 5 } $\mathrm{i}$ & $c_{\mathrm{i}}$ & $E_{\mathrm{i}}$ & $c_{\mathrm{i}}$ & $E_{\mathrm{i}}$ \\
\hline 1 & $3.399[-5]$ & $5.271[-1]$ & $2.015[-7]$ & $1.935[-4]$ \\
2 & $3.355[-5]$ & $1.641[-1]$ & $3.625[-5]$ & $5.599[-2]$ \\
3 & $1.846[-4]$ & $7.455[-1]$ & $2.828[-4]$ & $7.075[-1]$ \\
4 & $2.335[-4]$ & $2.172[0]$ & $1.352[-4]$ & $1.036[0]$ \\
5 & $1.200[-3]$ & $4.991[0]$ & $1.200[-3]$ & $1.221[0]$ \\
6 & $4.910[-3]$ & $1.200[1]$ & $2.797[-3]$ & $1.864[0]$ \\
7 & $7.110[-3]$ & $5.437[1]$ & $6.804[-3]$ & $2.160[0]$ \\
8 & $4.048[-2]$ & $1.310[2]$ & $5.229[-2]$ & $6.549[0]$ \\
\hline
\end{tabular}

Notes. The units of $c_{\mathrm{i}}$ and $E_{\mathrm{i}}$ are $\mathrm{cm}^{3} \mathrm{~s}^{-1} \mathrm{~K}^{3 / 2}$ and eV, respectively. Numbers in the square brackets are powers of 10 .

low-lying DR resonance strengths should be responsible for these discrepancies. At temperatures beyond $3 \times 10^{6} \mathrm{~K}$, where collisionally ionized plasmas form, and higher, the rate coefficients of the $\Delta N=0 \mathrm{DR}$ resonances are dominated by the recombination via $2 \mathrm{~s} 2 \mathrm{p}^{6}\left[{ }^{2} \mathrm{~S}_{1 / 2}\right] \mathrm{nl}(n \geq 7)$ intermediate states. Agreement within $20 \%$ is achieved between the experimentally derived and the theoretically calculated data.

The experimentally derived plasma recombination rate coefficients and the FAC calculated values were parameterized so that the plasma modelers and astrophysicists can use our data more easily. As a fit function, we used the following the expression:

$\alpha\left(T_{\mathrm{e}}\right)=T_{\mathrm{e}}^{-3 / 2} \sum_{i=1}^{8} c_{\mathrm{i}} \cdot \exp \left(-\frac{E_{\mathrm{i}}}{k T_{\mathrm{e}}}\right)$.

The resulting parameters $c_{\mathrm{i}}$ and $E_{\mathrm{i}}$ are listed in Table 1. The fit reproduces the plasma recombination rate coefficient in the temperature range of $10^{3}-10^{8} \mathrm{~K}$ within an uncertainty of below $0.5 \%$.

\section{Conclusion}

We measured the $\Delta N=0$ DR rate coefficients for the F-like nickel ions at the heavy-ion storage ring CSRm at the Institute of Modern Physics in Lanzhou, China. Fully relativistic theoretical calculations for the related processes by the FAC code have also been presented and compared with the measured spectrum in detail. Reasonable agreement is achieved between the measured recombination rate coefficients and theoretically calculated results. However, the prediction of the low-lying DR resonance positions, in particular the recombination via the $2 s 2 p^{6}\left[{ }^{2} S_{1 / 2}\right] 61$ intermediate states, still needs to be improved for the present theoretical model. The calculation significantly underestimates the resonance strengths at low collision energies. Temperature dependent plasma recombination rate coefficients have been derived from the measured DR rate coefficients and compared with the present FAC calculation as well as the available data from literature. The difference between the measurement and calculation is found to be less than $20 \%$ for temperatures where $\mathrm{Ni}^{19+}$ forms in a collisionally ionized plasma.
The calculation significantly differs from the experimental results around temperatures where $\mathrm{Ni}^{19+}$ ions form in photoionized plasmas. This is due to the difficulty in calculating the low-energy DR resonances with sufficient accuracy, which are subject to strong correlation and numerical cancelation effects. Our experimentally measured rate coefficient can serve as a benchmark for use in astrophysical and laboratory plasma modeling.

Acknowledgements. This work is partly supported by the National Key R\&D Program of China under grant No. 2017YFA0402300; the National Natural Science Foundation of China through grant No. U1732133; the Strategic Priority Research Program of the Chinese Academy of Sciences, grants No. XDPB09-02; and No. XDB21030300 and the Key Research Program of Frontier Sciences, CAS, grant No. QYZDY-SSW-SLH006. W. Wen acknowledges the support from the Youth Innovation Promotion Association of the Chinese Academy of Sciences. S. Schippers gratefully acknowledges support from the CAS President's International Fellowship Initiative (PIFI). The authors would like to thank the CSR accelerator staff for their technical support during the experiment. The discussion with A. Wolf and M. Lestinsky is greatly acknowledged.

\section{References}

Asplund, M., Grevesse, N., Sauval, A. J., \& Scott, P. 2009, ARA\&A, 47, 481 Beiersdorfer, P. 2003, ARA\&A, 41, 343

Bethe, H., \& Salpeter, E. 1957, Quantum Mechanics of One- and Two-Electron Systems (Springer)

Beyer, H. F., \& Shevelko, V. P. 2003, Series in Atomic and Molecular Physics : Introduction to the Physics of Highly Charged Ions (IOP Publishing Ltd)

Böhm, S., Schippers, S., Shi, W., et al. 2001, Phys. Rev. A, 64, 032707

Brandau, C., Kozhuharov, C., Lestinsky, C., et al. 2015, Phys. Scr., T166, 014022 Burgess, A. 1964, ApJ, 139, 776

Danared, H. 1993, Nucl. Instrum. Methods. A, 335, 397

Doschek, G. A., \& Feldman, U. 2010, J. Phys. B: At. Mol. Opt. Phys., 43, 232001

Feldman, U., \& Laming, J. M. 2000, Phys. Scr., 61, 222

Fogle, M., Badnell, N., Eklöw, N., Mohamed, T., \& Schuch, R. 2003a, A\&A, 409, 781

Fogle, M., Eklöw, N., Lindroth, E., et al. 2003b, J. Phys. B: At. Mol. Opt. Phys., 36,2563

Fogle, M., Badnell, N. R., Glans, P., et al. 2005, A\&A, 442, 757

Group, T. 1980, Plasma Phys., 22, 851

Gu, M. F. 2003, ApJ, 590, 1131

Gu, M. F. 2008, Can. J. Phys., 86, 675

Gwinner, G., Hoffknecht, A., Bartsch, T., et al. 2000, Phys. Rev. Lett., 84, 4822

Hahn, Y. 1993, J. Quant. Spectrosc. Radiat. Transfer, 49, 81

Henry, R. 1984, ApJ, 281, 644

Huang, Z. K., Wen, W. Q., Wang, H. B., et al. 2015, Phys. Scr., T166, 14023

Huang, Z. K., Wen, W. Q., Xu, X., et al. 2018, ApJS, 235, 2

Jacobs, V., Davis, J., Rogerson, J., et al. 1980, ApJ, 239, 1119

Kramida, A., Ralchenko, Yu., Reader, J., \& NIST ASD Team 2018, NIST Atomic Spectra Database (ver. 5.6.1), Available: https: //physics.nist. gov/asd [2019, April 10]. National Institute of Standards and Technology, Gaithersburg, MD

Lampert, A., Wolf, A., Habs, D., et al. 1996, Phys. Rev. A, 53, 1413

Mazzotta, P., Mazzitelli, G., Colafrancesco, S., \& Vittorio, N. 1998, A\&AS, 133, 403

Müller, A. 2008, Adv. At. Mol. Opt. Phy., 55, 293

Orban, I., Altun, Z., Källberg, A., et al. 2009, A\&A, 498, 909

Phillips, K. 2004, ApJ, 605, 921

Phillips, K., Fawcett, B., Kent, B., et al. 1982, ApJ, 256, 774

Savin, D. W. 2007, J. Phys. Conf. Ser., 88, 012071

Savin, D. W., \& Laming, J. M. 2002, ApJ, 566, 1166

Savin, D. W., Bartsch, T., Chen, M., et al. 1997, ApJ, 489, L115

Savin, D. W., Kahn, S., Linkemann, J., et al. 1999, ApJS, 123, 687

Schippers, S. 2009, J. Phys. Conf. Ser., 163, 012001

Schippers, S. 2015, Nucl. Instrum. Methods B, 350, 61

Schippers, S., Bartsch, T., Brandau, C., et al. 2000, Phys. Rev. A, 62, 022708

Schippers, S., Schnell, M., Brandau, C., et al. 2004, A\&A, 421, 1185

Schuch, R., \& Böhm, S. 2007, J. Phys. Conf. Ser., 88, 012002

Shull, J. M., \& Van Steenberg, M. 1982, ApJS, 48, 95

Wang, S., Xu, X., Huang, Z., et al. 2018, ApJ, 862, 134

Wen, W. Q., Ma, X., Xu, W. Q., et al. 2013, Nucl. Instrum. Methods B, 317, 731

Zatsarinny, O., Gorczyca, T., Fu, J., et al. 2006, A\&A, 447, 379 
S.-X. Wang et al.: Dielectronic recombination rate coefficients of fluorine-like nickel

\section{Appendix A: Additional table}

Table A.1. Comparison of the FAC calculated and CSRm measured resonance energies $E_{d}$ and strengths $S_{d}$.

\begin{tabular}{|c|c|c|c|c|}
\hline \multirow[t]{2}{*}{ Intermediate configurations } & \multicolumn{2}{|c|}{$E_{d}(\mathrm{eV})$} & \multicolumn{2}{|c|}{$S_{d}(\mathrm{eV})$} \\
\hline & FAC & Experiment $^{(b)}$ & FAC & Experiment $^{(b),(c)}$ \\
\hline $2 \mathrm{~s} 2 \mathrm{p}^{6}\left[{ }^{2} \mathrm{~S}_{1 / 2}\right] 6 \mathrm{~s}(\mathrm{~J}=1)$ & 0.1282 & $0.086 \pm 0.001$ & 635.5 & $1077.8 \pm 6.4$ \\
\hline $2 \mathrm{~s}^{2} 2 \mathrm{p}^{5}\left[{ }^{2} \mathrm{P}_{1 / 2}\right] 17 \mathrm{~s}$ & $0.3453^{(a)}$ & $0.330 \pm 0.006$ & 9.55 & $19.5 \pm 3.4$ \\
\hline $2 s^{2} 2 p^{5}\left[{ }^{2} P_{1 / 2}\right] 17 p$ & $0.5306^{(a)}$ & $0.528 \pm 0.003$ & 22.63 & $43.6 \pm 3.2$ \\
\hline $2 \mathrm{~s} 2 \mathrm{p}^{6}\left[{ }^{2} \mathrm{~S}_{1 / 2}\right] 6 \mathrm{~s}(\mathrm{~J}=0)$ & 0.7472 & & 54.54 & \\
\hline $2 \mathrm{~s}^{2} 2 \mathrm{p}^{5}\left[^{2} \mathrm{P}_{1 / 2}\right] 17 \mathrm{~d}$ & $0.7476^{(a)}$ & & 34.71 & \\
\hline Blend & $0.7474^{(a)}$ & $0.719 \pm 0.002$ & 89.25 & $95.2 \pm 3.1$ \\
\hline $2 \mathrm{~s}^{2} 2 \mathrm{p}^{5}\left[{ }^{2} \mathrm{P}_{1 / 2}\right] 171(l \geq f)$ & $0.8590^{(a)}$ & $0.846 \pm 0.001$ & 89.39 & $160.2 \pm 3.1$ \\
\hline (d) $2 \mathrm{~s}^{2} 2 \mathrm{p}^{5}\left[{ }^{2} \mathrm{P}_{1 / 2}\right] 18 \mathrm{~s}$ & $2.265^{(a)}$ & $2.267 \pm 0.019$ & 1.217 & $12.2 \pm 3.0$ \\
\hline (d) $2 \mathrm{~s}^{2} 2 \mathrm{p}^{5}\left[{ }^{2} \mathrm{P}_{1 / 2}\right] 18 \mathrm{p}$ & $2.421^{(a)}$ & $2.435 \pm 0.024$ & 4.158 & $9.7 \pm 3.0$ \\
\hline $2 \mathrm{~s}^{2} 2 \mathrm{p}^{5}\left[{ }^{2} \mathrm{P}_{1 / 2}\right] 181(l \geq p)$ & $2.664^{(a)}$ & $2.673 \pm 0.004$ & 36.94 & $57.4 \pm 2.9$ \\
\hline (d) $2 \mathrm{~s}^{2} 2 \mathrm{p}^{5}\left[{ }^{2} \mathrm{P}_{1 / 2}\right] 19 \mathrm{~s}$ & $3.886^{(a)}$ & $3.770 \pm 0.065$ & 0.599 & $4.4 \pm 3.0$ \\
\hline (d) $2 \mathrm{~s}^{2} 2 \mathrm{p}^{5}\left[{ }^{2} \mathrm{P}_{1 / 2}\right] 19 \mathrm{p}$ & $4.018^{(a)}$ & $3.968 \pm 0.030$ & 2.122 & $9.7 \pm 2.9$ \\
\hline $2 \mathrm{~s}^{2} 2 \mathrm{p}^{5}\left[{ }^{2} \mathrm{P}_{1 / 2}\right] 191(l \geq p)$ & $4.224^{(a)}$ & $4.229 \pm 0.010$ & 19.69 & $39.8 \pm 2.9$ \\
\hline $2 \mathrm{~s} 2 \mathrm{p}^{6}\left[{ }^{2} \mathrm{~S}_{1 / 2}\right] 6 \mathrm{p}_{1 / 2}$ & $4.409^{(a)}$ & $4.388 \pm 0.010$ & 30.42 & $36.9 \pm 3.6$ \\
\hline $2 \mathrm{~s} 2 \mathrm{p}^{6}\left[{ }^{2} \mathrm{~S}_{1 / 2}\right] 6 \mathrm{p}_{3 / 2}(\mathrm{~J}=2)$ & 4.831 & $4.750 \pm 0.023$ & 26.29 & $19.3 \pm 6.8$ \\
\hline $2 \mathrm{~s} 2 \mathrm{p}^{6}\left[{ }^{2} \mathrm{~S}_{1 / 2}\right] 6 \mathrm{p}_{3 / 2}(\mathrm{~J}=1)$ & 5.007 & $4.931 \pm 0.012$ & 38.62 & $78.0 \pm 5.0$ \\
\hline $2 s^{2} 2 p^{5}\left[{ }^{2} P_{1 / 2}\right] 201$ & $5.533^{(a)}$ & $5.568 \pm 0.013$ & 14.5 & $23.5 \pm 2.9$ \\
\hline $2 s^{2} 2 p^{5}\left[{ }^{2} \mathrm{P}_{1 / 2}\right] 211$ & $6.682^{(a)}$ & $6.707 \pm 0.021$ & 10.33 & $17.3 \pm 3.2$ \\
\hline $2 s^{2} 2 p^{5}\left[{ }^{2} P_{1 / 2}\right] 22 l$ & $7.678^{(a)}$ & $7.675 \pm 0.032$ & 7.792 & $12.2 \pm 3.2$ \\
\hline $2 s^{2} 2 p^{5}\left[{ }^{2} P_{1 / 2}\right] 231$ & $8.547^{(a)}$ & $8.568 \pm 0.037$ & 6.108 & $11.0 \pm 3.2$ \\
\hline $2 \mathrm{~s}^{2} 2 \mathrm{p}^{5}\left[{ }^{2} \mathrm{P}_{1 / 2}\right] 241$ & $9.309^{(a)}$ & $9.301 \pm 0.029$ & 4.922 & $9.4 \pm 3.0$ \\
\hline $2 \mathrm{~s} 2 \mathrm{p}^{6}\left[{ }^{2} \mathrm{~S}_{1 / 2}\right] 6 \mathrm{~d}_{3 / 2}$ & $9.714^{(a)}$ & & 29.11 & \\
\hline $2 \mathrm{~s} 2 \mathrm{p}^{6}\left[{ }^{2} \mathrm{~S}_{1 / 2}\right] 6 \mathrm{~d}_{5 / 2}(\mathrm{~J}=3)$ & 9.792 & & 25.35 & \\
\hline Blend & $9.750^{(a)}$ & $9.644 \pm 0.009$ & 54.46 & $58.0 \pm 3.4$ \\
\hline $2 \mathrm{~s}^{2} 2 \mathrm{p}^{5}\left[^{2} \mathrm{P}_{1 / 2}\right] 25 \mathrm{l}$ & $9.981^{(a)}$ & $9.869 \pm 0.034$ & 4.052 & $14.0 \pm 3.4$ \\
\hline $2 \mathrm{~s} 2 \mathrm{p}^{6}\left[{ }^{2} \mathrm{~S}_{1 / 2}\right] 6 \mathrm{~d}_{5 / 2}(\mathrm{~J}=2)$ & 10.25 & $10.19 \pm 0.018$ & 17.12 & $20.9 \pm 2.6$ \\
\hline $2 \mathrm{~s}^{2} 2 \mathrm{p}^{5}\left[{ }^{2} \mathrm{P}_{1 / 2}\right] 261$ & $10.58^{(a)}$ & $10.58 \pm 0.041$ & 3.392 & $9.1 \pm 2.6$ \\
\hline $2 s^{2} 2 p^{5}\left[{ }^{2} P_{1 / 2}\right] 271$ & $11.11^{(a)}$ & $11.16 \pm 0.054$ & 2.879 & $7.2 \pm 2.6$ \\
\hline $2 \mathrm{~s}^{2} 2 \mathrm{p}^{5}\left[{ }^{2} \mathrm{P}_{1 / 2}\right] 281$ & $11.58^{(a)}$ & $11.58 \pm 0.057$ & 2.472 & $7.1 \pm 2.6$ \\
\hline $2 s^{2} 2 p^{5}\left[{ }^{2} P_{1 / 2}\right] 291$ & $12.00^{(a)}$ & $11.97 \pm 0.029$ & 2.142 & $13.7 \pm 2.6$ \\
\hline $2 \mathrm{~s} 2 \mathrm{p}^{6}\left[{ }^{2} \mathrm{~S}_{1 / 2}\right] 6 \mathrm{f}$ & $12.30^{(a)}$ & & 105.5 & \\
\hline $2 s^{2} 2 p^{5}\left[{ }^{2} P_{1 / 2}\right] 301$ & $12.40^{(a)}$ & & 1.873 & \\
\hline Blend & $12.30^{(a)}$ & $12.23 \pm 0.004$ & 107.4 & $111.4 \pm 2.5$ \\
\hline $2 \mathrm{~s} 2 \mathrm{p}^{6}\left[{ }^{2} \mathrm{~S}_{1 / 2}\right] 61(l=g, h)$ & $12.55^{(a)}$ & & 162 & \\
\hline $2 s^{2} 2 p^{5}\left[{ }^{2} P_{1 / 2}\right] 311$ & $12.74^{(a)}$ & & 1.649 & \\
\hline Blend & $12.56^{(a)}$ & $12.60 \pm 0.002$ & 163.6 & $178.8 \pm 2.6$ \\
\hline $2 s^{2} 2 p^{5}\left[{ }^{2} P_{1 / 2}\right] 321$ & $13.06^{(a)}$ & $12.97 \pm 0.046$ & 1.461 & $9.1 \pm 2.5$ \\
\hline $2 \mathrm{~s} 2 \mathrm{p}^{6}\left[{ }^{2} \mathrm{~S}_{1 / 2}\right] 7 \mathrm{~s}$ & $41.12^{(a)}$ & $41.49 \pm 0.65$ & 1.2 & $0.7 \pm 1.1$ \\
\hline $2 \mathrm{~s} 2 \mathrm{p}^{6}\left[{ }^{2} \mathrm{~S}_{1 / 2}\right] 7 \mathrm{p}$ & $43.90^{(a)}$ & $44.16 \pm 0.08$ & 3.638 & $6.2 \pm 1.0$ \\
\hline $2 \mathrm{~s} 2 \mathrm{p}^{6}\left[{ }^{2} \mathrm{~S}_{1 / 2}\right] 7 \mathrm{~d}$ & $47.08^{(a)}$ & $47.30 \pm 0.05$ & 7.183 & $10.5 \pm 1.1$ \\
\hline $2 \mathrm{~s} 2 \mathrm{p}^{6}\left[{ }^{2} \mathrm{~S}_{1 / 2}\right] 71(l \geq d)$ & $48.69^{(a)}$ & $48.91 \pm 0.04$ & 35.28 & $42.5 \pm 16$ \\
\hline $2 \mathrm{~s} 2 \mathrm{p}^{6}\left[{ }^{2} \mathrm{~S}_{1 / 2}\right] 8 \mathrm{~s}$ & $67.16^{(a)}$ & $67.64 \pm 0.34$ & 0.735 & $1.1 \pm 0.8$ \\
\hline $2 \mathrm{~s} 2 \mathrm{p}^{6}\left[{ }^{2} \mathrm{~S}_{1 / 2}\right] 8 \mathrm{p}$ & $69.00^{(a)}$ & $69.24 \pm 0.23$ & 2.526 & $1.8 \pm 0.8$ \\
\hline $2 \mathrm{~s} 2 \mathrm{p}^{6}\left[{ }^{2} \mathrm{~S}_{1 / 2}\right] 8 \mathrm{~d}$ & $71.09^{(a)}$ & $71.33 \pm 0.11$ & 4.174 & $4.6 \pm 1.0$ \\
\hline $2 \mathrm{~s} 2 \mathrm{p}^{6}\left[{ }^{2} \mathrm{~S}_{1 / 2}\right] 81(l \geq d)$ & $72.24^{(a)}$ & $72.57 \pm 0.08$ & 24.28 & $22.6 \pm 1.4$ \\
\hline (d) $2 \mathrm{~s} 2 \mathrm{p}^{6}\left[{ }^{2} \mathrm{~S}_{1 / 2}\right] 9 \mathrm{~s}$ & $84.82^{(a)}$ & $85.02 \pm 0.18$ & 0.458 & $2.3 \pm 0.9$ \\
\hline $2 \mathrm{~s} 2 \mathrm{p}^{6}\left[{ }^{2} \mathrm{~S}_{1 / 2}\right] 9 \mathrm{p}$ & $86.10^{(a)}$ & $86.86 \pm 0.09$ & 1.53 & $4.8 \pm 0.9$ \\
\hline $2 \mathrm{~s} 2 \mathrm{p}^{6}\left[{ }^{2} \mathrm{~S}_{1 / 2}\right] 9 \mathrm{~d}$ & $87.56^{(a)}$ & $88.17 \pm 0.61$ & 2.67 & $4.9 \pm 5.6$ \\
\hline
\end{tabular}

Notes. The uncertainties here are only the errors resulting from our resonance fits. The uncertainties from the absolute measurement are not included. ${ }^{(a)}$ Weighted energy: $E_{d}=\sum E_{d} \mathrm{~S}_{d} / \sum \mathrm{S}_{d}{ }^{(b)}$ Standard error from the fit at $1 \sigma$ confidence level only. ${ }^{\left({ }^{(}\right)}$The experimental measured rate coefficients have a systematic uncertainty of about $30 \%$. ${ }^{(d)}$ The fitted results for these weak resonances are less reliable. 
A\&A 627, A171 (2019)

Table A.1. continued.

\begin{tabular}{|c|c|c|c|c|}
\hline \multirow[t]{2}{*}{ Intermediate configurations } & \multicolumn{2}{|c|}{$E_{d}(\mathrm{eV})$} & \multicolumn{2}{|c|}{$S_{d}(\mathrm{eV})$} \\
\hline & FAC & Experiment $^{(b)}$ & FAC & Experiment $^{(b),(c)}$ \\
\hline $2 \mathrm{~s} 2 \mathrm{p}^{6}\left[{ }^{2} \mathrm{~S}_{1 / 2}\right] 91(l \geq d)$ & $88.37^{(a)}$ & $88.73 \pm 0.04$ & 18.53 & $18.4 \pm 5.6$ \\
\hline (d) $2 \mathrm{~s} 2 \mathrm{p}^{6}\left[{ }^{2} \mathrm{~S}_{1 / 2}\right] 10 \mathrm{~s}$ & $97.33^{(a)}$ & $97.41 \pm 3.4$ & 0.348 & $1.4 \pm 12$ \\
\hline (d) $2 \mathrm{~s} 2 \mathrm{p}^{6}\left[{ }^{2} \mathrm{~S}_{1 / 2}\right] 10 \mathrm{p}$ & $98.26^{(a)}$ & $97.71 \pm 3.6$ & 1.129 & $0.4 \pm 12$ \\
\hline (d) $2 \mathrm{~s} 2 \mathrm{p}^{6}\left[{ }^{2} \mathrm{~S}_{1 / 2}\right] 10 \mathrm{~d}$ & $99.32^{(a)}$ & $99.70 \pm 3.1$ & 1.987 & $1.3 \pm 0.7$ \\
\hline $2 \mathrm{~s} 2 \mathrm{p}^{6}\left[{ }^{2} \mathrm{~S}_{1 / 2}\right] 101(l \geq d)$ & $99.91^{(a)}$ & $100.32 \pm 0.05$ & 15.77 & $18.4 \pm 6.6$ \\
\hline (d) $2 \mathrm{~s} 2 \mathrm{p}^{6}\left[{ }^{2} \mathrm{~S}_{1 / 2}\right] 11 \mathrm{~s}$ & $106.5^{(a)}$ & & 0.29 & \\
\hline (d) $2 \mathrm{~s}^{2} \mathrm{p}^{6}\left[{ }^{2} \mathrm{~S}_{1 / 2}\right] 11 \mathrm{p}$ & $107.2^{(a)}$ & & 0.915 & \\
\hline (d) $2 \mathrm{~s} 2 \mathrm{p}^{6}\left[{ }^{2} \mathrm{~S}_{1 / 2}\right] 11 \mathrm{~d}$ & $108.0^{(a)}$ & & 1.607 & \\
\hline $2 \mathrm{~s} 2 \mathrm{p}^{6}\left[{ }^{2} \mathrm{~S}_{1 / 2}\right] 111(l \geq d)$ & $108.5^{(a)}$ & $108.9 \pm 0.21$ & 14 & $20.1 \pm 2.6$ \\
\hline
\end{tabular}

